PROTEOMIC ANALYSIS

\section{Protein modification analysis in depth}

Nat. Commun., https://doi.org/10.1038/s41467018-04957-4 (2018)

In eukaryotes, small ubiquitin-like modifier (SUMO) is a protein family with four different isoforms (S1 to S4) that has enzymes dedicated to conjugating SUMO to target proteins. SUMOylation impacts enzyme location and function and has been implicated in cell division, nuclear transport, and transcriptional regulation. In a new report, investigators describe a method to isolate S2 and S3 conjugated peptides, which does not rely on engineered cell lines or animals carrying a tagged variant of SUMO to facilitate enrichment. The authors identified more than 14,000 sites in human cell culture and nearly 2000 across eight different mouse tissues. In cell culture, a much larger fraction of SUMO2/3 pools were conjugated to targets and is consistent with other reports of high SUMOylation levels in rapidly dividing tissues. Brain had the fewest sites of any mouse tissue and is surprising given that SUMO has been implicated in some neuropathologies. This dataset will serve as a reference of SUMOylation in normal/healthy tissues. $C N$ https://doi.org/10.1038/s41684-018-0123-7

BONE DEVELOPMENT

\section{Playing catch-up}

PLoS Biol, https://doi.org/10.1371/journal. pbio.2005086 (2018).

When an organism suffers non-symmetric damage during development, the impacted tissue must repair itself and grow disproportionally quicker in order to regain proper size and proportions. Authors of a recent study present a novel model for studying this process and extend our understanding of long bone development in fetal mice. In the report, investigators delivered an inducible cartilage-specific cell cycle repressor that blocked cell division in a fraction of chondrocytes on the left side, leaving the right side as the control. As compensation, unmodified left-side chondrocytes increased proliferation and there was also a net increase in extracellular matrix deposition. Interestingly, there was a mild decrease in systemic growth, which authors speculate was a result of communication between impacted chondrocytes and the placenta. Such a decrease may facilitate the catch-up process.

\section{NEUROPHYSIOLOGY}

\section{Mapping behaviors to descending neurons}

Elife, https://doi.org/10.7554/eLife.34275 (2018).

In vertebrates and invertebrates, environmental information is processed in the brain and behavioral signals passed through descending neurons to regulatory centers that are proximal to effectors i.e. muscles. For Drosophila, only $\sim 1100$ neurons relay information to the ventral nerve cord that evokes the broad array of responses observed. To date, there is no high throughput way to relate specific descending neurons with a given behavior in flies. A new study remedies this with a technique to map the function of individual descending neurons. Using optogenetics, authors activated individual descending neurons and then employed an automated behavior classification system to analyze video captured data and assign a regulated behavior. Investigators classified between a third and a half of all descending neurons. The data revealed redundancy with many of the neurons eliciting similar behaviors that were stereotypical.

https://doi.org/10.1038/s41684-018-0125-5

\section{GENETIC ENGINEERING}

\section{New technique for knock-in mice}

Nat. Biotechnol., https://doi.org/10.1038/nbt.4166 (2018).

Targetable nucleases have made genetic engineering of animals for research a much more tractable endeavor. But, efficient transfer of large insertion products remains challenging and inefficient, despite several recent innovations. To date, most reported techniques transformed zygotes for generation of engineered mouse lines. Authors of a new manuscript took a different approach and introduced CRISPR/Cas9 reagents to two-cell zygotes so as to take advantage of the extended G2 phase-post-DNA replication and pre-mitosis-to increase the time during which recombination could occur. Investigators also used a streptavidin tagged Cas9 in combination with a biotinylated repair template to increase efficient construct interaction and thereby improve recombination. With these alterations, transformation efficiency improved several fold over conventional methods.

https://doi.org/10.1038/s41684-018-0126-4

Ellen P. Neff and Clark Nelson

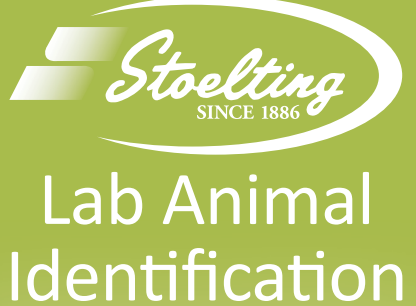

SMALL ANIMAL
EARTAGS AND MARKERS

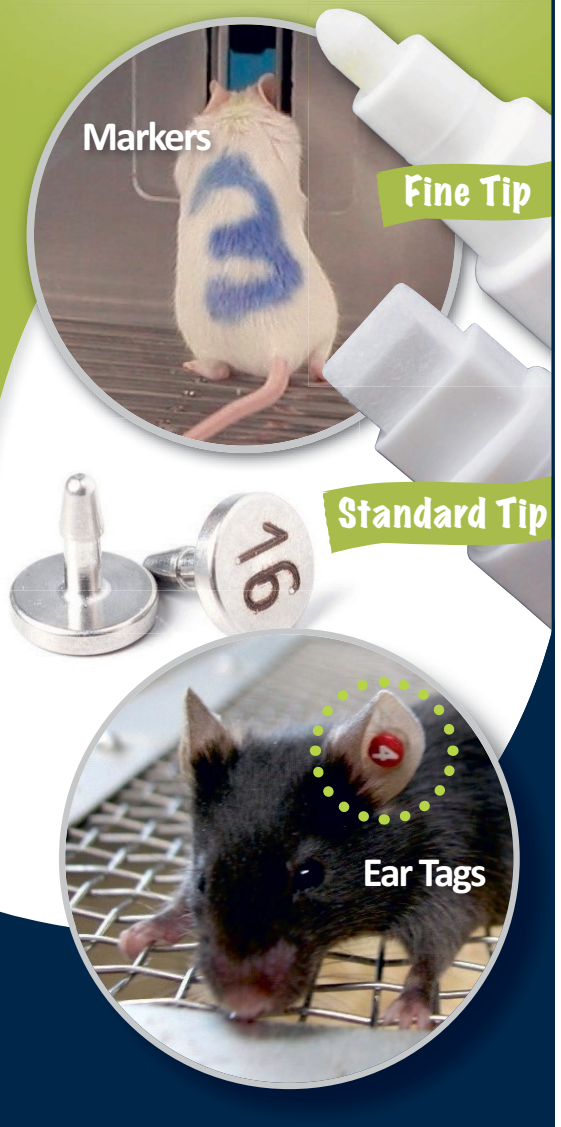

Ear Tag and Marker Features:

- Quick and easy identification

- Long-lasting

- Nontoxic, non-hazardous

- Multiple colors options available

Contact Stoelting for details! Visit www.StoeltingCo.com or email us at https://doi.org/10.1038/s41684-018-0124-6 